\title{
Effect of Heat Treatment Process on the Structure and Properties of $\mathrm{Nano}^{-\mathrm{TiO}_{2}}$
}

\author{
R. Q. Gao†, Y. R. Huang, D. Liu and G. T. Li
}

School of Environmental and Municipal Engineering, North China University of Water Resources and Electric Power, Zhengzhou 450045, China

†Corresponding author: R. Q. Gao; 15838335721@163.com; gaoruqin@ncwu.edu.cn

Nat. Env. \& Poll. Tech.

Website: www.neptjournal.com

Received: 11-02-2020

Revised: $02-03-2020$

Accepted: 02-05-2020

\section{Key Words:}

Sol-gel method

Titanium dioxide

Sintering temperature

Crystal-type transformation

Photocatalysis

\begin{abstract}
Nano- $\mathrm{TiO}_{2}$ was prepared with butyl titanate as a precursor by sol-gel method. The samples were analysed by TG-DTA, X-ray diffraction, TEM and so on to assess the effects of different temperatures on the crystal structure, grain size, and microstructure of nano- $\mathrm{TiO}_{2}$. Meanwhile, the catalytic effect of heat treatment temperature on the degradation performance of $\mathrm{TiO}_{2}$ to methyl orange was investigated. The dynamic process of grain growth was preliminarily analysed by Eastman's particle growth theory. The result shows that $\mathrm{TiO}_{2}$ particle size gradually increases with the heat treatment temperature. At 450 to $550^{\circ} \mathrm{C}$, the grain is mainly anatase phase, a mixture of anatase and rutile phase was found at $650^{\circ} \mathrm{C}$ (mass ratio $A: R=9: 1$ ), and the degradation rate of nano- $\mathrm{TiO}_{2}$ on methyl orange reaches $97.75 \%$. When the calcination temperature exceeds $850^{\circ} \mathrm{C}, \mathrm{TiO}_{2}$ particles almost entirely are composed of rutile phase, and the photocatalytic activity decreases significantly. At $730^{\circ} \mathrm{C}$, half of the crystalline $\mathrm{TiO}_{2}$ is transformed from anatase to rutile form. The apparent activation energies of the anatase and rutile crystals of nano- $\mathrm{TiO}_{2}$ are $18.15 \mathrm{~kJ} / \mathrm{mol}$ and $42.56 \mathrm{~kJ} / \mathrm{mol}$, and the fastest grain growth occurs at $546^{\circ} \mathrm{C}$ and $1280^{\circ} \mathrm{C}$ respectively.
\end{abstract}

\section{INTRODUCTION}

Photocatalytic oxidation technology has the prominent characteristics of low energy consumption, strong oxidation ability, mild reaction conditions, and reduced secondary pollution when removing pollutants (Liu et al. 2013, Chekem et al. 2019, Varnagiris et al. 2019). It has the potential for broad application in sewage treatment and air purification (Reddy et al. 2017, Al-Mamuna et al. 2019, Srikanth et al. 2017), and the development of nano- $\mathrm{TiO}_{2}$ has become an active area of scientific research (Zhang et al. 2014). The sol-gel method can be used to prepare nanometric catalysts with high purity, uniform particle size, and high chemical activity at low temperature, and the reaction conditions are easy to control and the preparation process is relatively simple (Hu et al. 2010, Hakki et al. 2019, Wei et al. 2018). Therefore, the sol-gel method has attracted more attention due to its significant advantages in film formation and doping modification; however, to prepare $\mathrm{TiO}_{2}$ with high photocatalytic activity, powders with uniform grain size and a low degree of agglomeration are required. Among them, the heat treatment process is one of the important means to promote the transition from amorphous to the crystalline state and adjust the grain size (Liao et al. 2011, Tong et al. 2018, Sun et al. 2017). In this study, $\mathrm{TiO}_{2}$ particles were synthesised by sol-gel method, and the effects of heat treatment on the crystal structure, particle size, and crystal morphology of $\mathrm{TiO}_{2}$ particles were analysed by XRD and TEM, and the reasons for $\mathrm{TiO}_{2}$ phase transition during heat treatment were preliminarily analysed from the perspective of reaction kinetics, and the effect of heat treatment temperature on the photocatalytic performance of $\mathrm{TiO}_{2}$ has also been discussed.

\section{MATERIALS AND METHODS}

Material: Butyl titanate (Tianjin Comeo Chemical Reagent Co., Ltd), anhydrous ethanol (Tianjin Fuyu Fine Chemical Co., Ltd), glacial acetic acid (Xilong Chemical Co., Ltd), concentrated nitric acid (Kaifeng Huatong Chemical Co., Ltd), polyethylene glycol-4000 (Tianjin Komio Chemical Reagent Co., Ltd), and methyl orange (Tianjin Gold Platinum Orchid Fine Chemicals Ltd) were of analytical purity.

Sample preparation and characterization: At room temperature, $10 \mathrm{~mL}$ of butyl titanate was fully mixed with 10 $\mathrm{mL}$ of anhydrous ethanol as Solution A. $4 \mathrm{~mL}$ of deionised water, $20 \mathrm{~mL}$ of glacial acetic acid, $2 \mathrm{~mL}$ of concentrated nitric acid, and $10 \mathrm{~mL}$ of anhydrous ethanol were fully mixed Solution B. Under vigorous stirring, Solution B was added (drop-wise) into Solution A, then $0.5 \mathrm{~g}$ polyethylene glycol-4000 was added (drop-wise), the hydrolysis temperature was maintained at $40^{\circ} \mathrm{C}$, and then stirred for $2 \mathrm{~h}$ to ensure the sufficient chelation. The product was dried at a constant temperature $60^{\circ} \mathrm{C}$ for $24 \mathrm{~h}$ and ground into a fine powder. After calcination at different temperatures $(450$ 
to $850^{\circ} \mathrm{C}$ ) for $2 \mathrm{~h}, \mathrm{TiO}_{2}$ powder can be obtained by cooling in the furnace to room temperature.

Using $\mathrm{A}_{2} \mathrm{O}_{3}$ powder as reference material, the composite was subjected to TG-DTA thermal analysis by NETZSCH STA 499C integrated thermal analyser at a rate of $20^{\circ} \mathrm{C} / \mathrm{min}$ in the air; we then used a JEOL 2100 transmission electron microscope (Shimazu, Japan) to observe the microstructure of the composite. A Bruker D8-advance X-ray diffractometer (Germany) was used for phase analysis of the samples; and ASAP 2010 specific surface area and pore size analyser (Micromeritics Co., USA) was employed to determine the specific surface area and total pore volume.

Photocatalytic activity test: The prepared $100 \mathrm{mg} \mathrm{\textrm {TiO } _ { 2 }}$ (calcined sample at different temperatures and $P_{25}$ ) was added to the $200 \mathrm{~mL}$ methyl orange solution with a mass concentration of $10 \mathrm{mg} / \mathrm{L}$. After magnetic stirring in the dark for $30 \mathrm{~min}$, the adsorption and desorption of methyl orange on the surface of $\mathrm{TiO}_{2}$ particles reached equilibrium. The specimen was then irradiated with UV light for $2 \mathrm{~h}$ (the main wavelength is $254 \mathrm{~nm}$, the distance between the light source and the liquid surface is $80 \mathrm{~mm}$ ), taking $10 \mathrm{~mL}$ every $0.5 \mathrm{~h}$. After centrifugation, the supernatant was taken and the absorbance measured at the maximum absorption wavelength of methyl orange at $464 \mathrm{~nm}$ to allow calculation of the rate of degradation rate $\eta$ :

$$
\eta=\frac{A_{0}-A}{A_{0}} \times 100 \%
$$

Where, $A_{0}$ and $A$ are the absorbances of the reaction solution before and after a certain illumination time.

\section{RESULTS AND DISCUSSION}

\section{DTA-TG Analysis of the Sample}

Fig. 1 shows the TG-DTA curve of $\mathrm{TiO}_{2}$ after drying at $105^{\circ} \mathrm{C}$. It can be seen from the DTA curve that there is an endothermic peak between about $50^{\circ} \mathrm{C}$ and $100^{\circ} \mathrm{C}$, and the mass loss on the TG curve is about $5 \%$. This peak represents the process of water absorption and drainage within the material. There is an exothermic peak between $300^{\circ} \mathrm{C}$ and $400^{\circ} \mathrm{C}$, and the mass loss on the TG curve is about $12 \%$. This peak is caused by the exotherm caused by the combustion of organic matter in $\mathrm{TiO}_{2}$ prepared by the sol-gel method. It has been reported in the literature that the powders prepared from titanium alcohols exhibit a strong exothermic peak at 400 to $500^{\circ} \mathrm{C}$, which is the result of the transformation from amorphous $\mathrm{TiO}_{2}$ to anatase-type $\mathrm{TiO}_{2}$; however, the $\mathrm{TiO}_{2}$ prepared with butyl titanate in this study only shows a weak similar exothermic peak on the DTA curve. There is a mass loss of $3 \%$ on the TG curve between $750^{\circ} \mathrm{C}$ and $950^{\circ} \mathrm{C}$ (while the exothermic peak on the DTA curve is not obvious), as attributed to the phase transition of anatase $\mathrm{TiO}_{2}$ to rutile $\mathrm{TiO}_{2}$, indicating that the crystalline transition (anatase-rutile phase) is a slow, gradual process.

\section{XRD Analysis}

Fig. 2 illustrates the X-ray diffraction patterns of nano- $\mathrm{TiO}_{2}$ prepared at different temperatures. It can be seen from the figure that the samples treated at $450^{\circ} \mathrm{C}$ and $550^{\circ} \mathrm{C}$ have diffraction peaks near $2 \theta=25.3^{\circ}, 37.7^{\circ}, 48.0^{\circ}, 53.8^{\circ}, 55.1^{\circ}$, and $62.8^{\circ}$, corresponding to the (101), (004), (200), (105), (211) and (204) planes of anatase phase, respectively, indicating that the product is mainly composed of anatase phase; the diffraction peaks are wider than normal, indicating that the particles are small, and the grain development is irregular. Quite sharp diffraction peaks and weak rutile phases appear at $650^{\circ} \mathrm{C}$ (Daniyal et al. 2019, Zarhri et al. 2020). The characteristic diffraction peaks of the samples treated at $850^{\circ} \mathrm{C}$ and $950^{\circ} \mathrm{C}$ were $2 \theta=27.4^{\circ}, 36.0^{\circ}, 41.3^{\circ}, 54.3^{\circ}$, and $69.1^{\circ}$ corresponding to the (110), (101), (111), (211), and (301) planes of the rutile $\mathrm{TiO}_{2}$, respectively, indicating that the products are mainly rutile. The phase change temperature of XRD is the heat treatment temperature when half of the

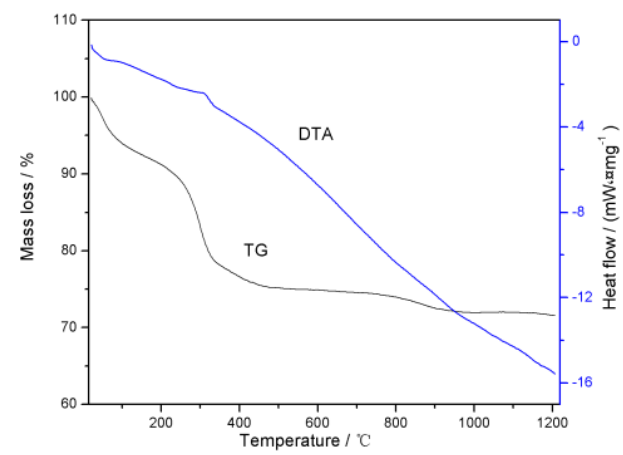

Fig. 1: TG-DTA patterns of $\mathrm{TiO}_{2}$.

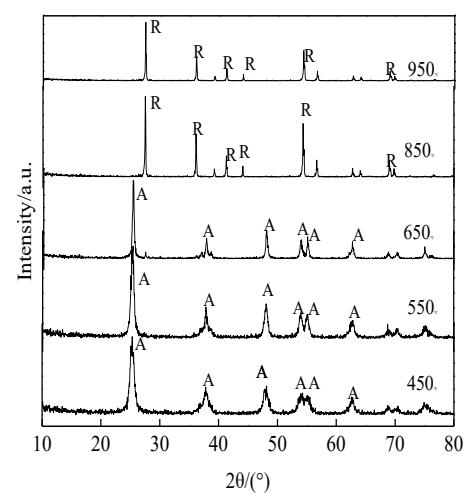

Fig. 2: XRD patterns of $\mathrm{TiO}_{2}$ at different sintering temperatures. 
anatase changes to rutile. As can be seen from Fig. 2, the phase change temperature is about $730^{\circ} \mathrm{C}$.

For this $\mathrm{TiO}_{2}$ the average particle size was estimated using the X-ray width method and the Scherrer formula. The mass percentage $X_{\mathrm{A}}$ of anatase phase in the samples was calculated thus (Zhang et al. 2017, Li et al. 2018): $X_{\mathrm{A}}=I_{\mathrm{A}} /$ $\left(I_{\mathrm{A}}+1.265 I_{\mathrm{R}}\right) \times 100 \%$, where, $I_{\mathrm{A}}$ is the diffraction intensity of the strongest characteristic peak of the anatase phase on the crystal plane (101), and $I_{\mathrm{R}}$ is the diffraction intensity of the strongest characteristic peak of the rutile phase on the crystal plane (110).

Table 1 gives the anatase and $\mathrm{TiO}_{2}$ contents at different heat treatment temperatures and the respective grain sizes: with the increase of the heat treatment temperature, in nano- $\mathrm{TiO}_{2}$, the anatase phase gradually changes to rutile phase. In the temperature range from 450 to $650^{\circ} \mathrm{C}$, due to the random grain orientation, there are more grain boundary defects and greater activity, structural relaxation was first produced in the grain boundary during the heating process, and the atomic rearrangement occurred, thus making the structure tend to become more orderly thus reducing the free energy of the grain boundary. Therefore, the apparent grain growth is slow within this temperature range; however, when the temperature rises to $650^{\circ} \mathrm{C}$, the structural relaxation process ends, the grain boundary migration process begins, and the grain size increases rapidly after entering the phase of grain growth and merger of large grains with small grains.

\section{TEM Analysis of Samples}

Fig. 3 shows the micro-morphology of nano- $\mathrm{TiO}_{2}$ at different calcining temperatures. The sample size at $450^{\circ} \mathrm{C}$ is small (about $10 \mathrm{~nm}$ ) and the particle boundaries are fuzzy, indicating that $\mathrm{TiO}_{2}$ has not yet formed complete grains at this calcination temperature, and most of the particles are in an amorphous state. The sample particles at $650^{\circ} \mathrm{C}$ are intact, the boundary is clear, and the particle size is about $20 \mathrm{~nm}$. The sample particle size at $950^{\circ} \mathrm{C}$ is coarse (at about $80 \mathrm{~nm}$ ), the boundary is clear, and the shape is not very regular. The test results are consistent with the analytical results of $\mathrm{TiO}_{2}$ grain size at different heat treatment temperatures.

\section{BET Analysis of Samples}

Table 2 provides the specific surface area and pore structure of the samples at different heat treatment temperatures. As seen from Table 2, the specific surface area and pore volume of the samples prepared at different calcination temperatures decreases with increasing calcination temperature because the increased calcination temperature leads to the gradual growth and refinement of $\mathrm{TiO}_{2}$ grains and the gradual

Table 1: Crystallite size and anatase content of samples at different heat treatment temperatures.

\begin{tabular}{|llllll|}
\hline Sintering temperature $/{ }^{\circ} \mathrm{C}$ & 450 & 550 & 650 & 850 & 950 \\
\hline Mass content $/ \%$ & 95.42 & 96.86 & 89.81 & 0.61 & 0.16 \\
Diameter/nm & 10.21 & 14.3 & 21.8 & 63.58 & 78.6 \\
\hline
\end{tabular}

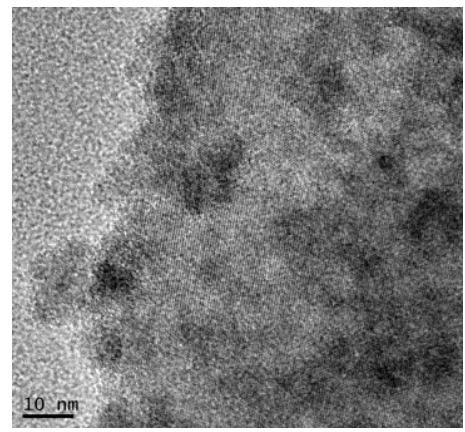

$450^{\circ} \mathrm{C}$

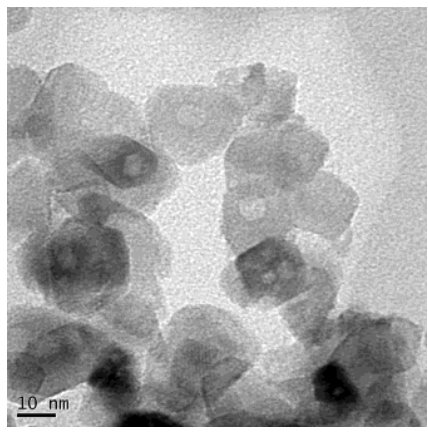

$650^{\circ} \mathrm{C}$

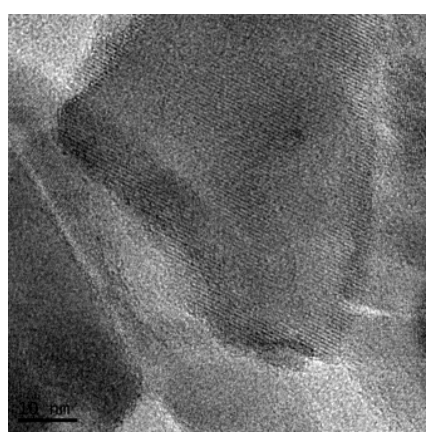

$950^{\circ} \mathrm{C}$

Fig. 3: TEM images of samples at different sintering temperatures.

Table 2: BET surface area and pore structure of samples at different heat treatment temperatures.

\begin{tabular}{|lllllll}
\hline Sintering temperature $/{ }^{\circ} \mathrm{C}$ & 450 & 550 & 650 & 850 & 950 \\
\hline $\mathrm{S}_{\mathrm{BET}} /\left(\mathrm{m}^{2} \mathrm{~g}^{-1}\right)$ & 223.5 & 167.8 & 83.1 & 35.7 & 3.4 \\
$\mathrm{~V}_{\text {pore }} /\left(\mathrm{cm}^{3} \mathrm{~g}^{-1}\right)$ & 0.21 & 0.18 & 0.16 & 0.09 & 0.03 \\
\hline
\end{tabular}


increase in grain size. When treated at $450^{\circ} \mathrm{C}$, nano- $\mathrm{TiO}_{2}$ had its maximum specific surface area and pore volume and, at thereat, the nano- $\mathrm{TiO}_{2}$ had not yet formed crystals, remaining in an amorphous state with small particles and uniform pores. In the heat-treatment temperature range from $550^{\circ} \mathrm{C}$ to $850^{\circ} \mathrm{C}$, the specific surface area and pore volume of $\mathrm{TiO}_{2}$ samples gradually decreased with the increase of temperature after roasting. This is because the increase in heat treatment temperature resulted in obvious growth of $\mathrm{TiO}_{2}$ grains. At the same time, because the small pores were subjected to greater pressure than the large pores during heat treatment, the pores formed by the accumulation of the original $\mathrm{TiO}_{2}$ particles collapsed or disappeared, gradually forming larger holes. When the temperature reached $950^{\circ} \mathrm{C}, \mathrm{TiO}_{2}$ particles grew to $78.6 \mathrm{~nm}$, the small particles merged to form larger particles, and the pore structure decreased in volume, so the specific surface area of the sample reached $3.4 \mathrm{~m}^{2} \mathrm{~g}^{-1}$ with a pore volume of $0.03 \mathrm{~cm}^{3} \cdot \mathrm{g}^{-1}$.

\section{Phase Change Kinetic Analysis}

According to Eastman particle growth theory:

$$
D_{\mathrm{t}}^{2}-D_{0}^{2}=\mathrm{kt}^{\mathrm{n}} \exp (-E / R T)
$$

Where, $D_{t}$ is the particle size of the grain at time $t(\mathrm{~nm})$; $D_{0}$ is the grain size at the initial time (nm); $k$ is constant; $n$ is the reaction index; $E$ represents the apparent activation energy $(\mathrm{J} / \mathrm{mol}) ; R$ is the ideal gas constant, and $T$ is the reaction temperature $(\mathrm{K})$; because the grain size of the initial stage of the preparation is very small, $D_{0} \approx 0$, and $t^{n}$ can also be treated as a constant when the heat treatment time is fixed. Let $K=k t^{n}$, then formula (2) can be transformed into:

$$
\begin{gathered}
D_{\mathrm{t}}^{2}=\mathrm{kt}^{\mathrm{n}} \exp (-E / R T)=\operatorname{Kexp}(-E / R T) \\
2 \ln D_{\mathrm{t}}=-E / R T+\ln K
\end{gathered}
$$

For (3) formula $\mathrm{d} D_{\mathrm{t}} / \mathrm{d} T$

$$
\mathrm{d} D_{\mathrm{t}} / \mathrm{d} T=K^{1 / 2} \exp (-E / 2 R T) \frac{E}{2 R T^{2}}
$$

Find the maximum value $\left(\mathrm{d}^{2} D_{t} / \mathrm{D} t^{2}=0\right)$ for equation (5), and we obtain the temperature at which the fastest grain growth occurred $T_{\text {fast }}$

$$
\frac{E}{4 R}=T_{\text {fast }}
$$

Plotting $2 \ln D$ v. $1 / T$ gives the straight line shown in Fig. $4,2 \ln D$ and $1 / T$ do not obey a single linear relationship because the $\mathrm{TiO}_{2}$ crystal type changes from anatase to rutile at around $730^{\circ} \mathrm{C}$, and Section $\mathrm{AB}$ of the curve is dominated by the anatase phase, while Section $\mathrm{BC}$ is dominated by the rutile phase. The lines of best-fit are $y=-4.3669 x+10.7697$ $\left(R^{2}=0.9252\right)$, and $y=-10.2403 x+17.2735\left(R^{2}=0.9667\right)$, respectively. From this, the apparent activation energies $E_{\text {a }}$ and $E_{\mathrm{r}}$ of anatase and rutile grain growth can be calculated (Table 3). The apparent activation energy of rutile $\mathrm{TiO}_{2}$ grain growth is larger than that of anatase-type growth. On the one hand, due to the early-stage growth, the $\mathrm{TiO}_{2}$ particle size is small, lattice defects are large, the particle growth is almost spontaneous, and the energy required is very small. Increased heat treatment temperature, fewer lattice defects (lattice development tends to be complete), and $\mathrm{TiO}_{2}$ grain growth difficulties, all cause the activation energy to increase; on the other hand, as the heat treatment temperature increases further, more of the smaller anatase $\mathrm{TiO}_{2}$ grains are transformed into rutile phase grains, and some fully-grown grains even undergo a phase transition. Fewer small grains are available for the continuous growth of anatase grains in the samples, so the activation energy of grain growth is greatly increased (Amora et al. 2019, Jithin et al. 2017).

\section{Effect of Heat Treatment Temperature on Photocatalytic Performance of Samples}

Fig. 5 demonstrates the heat treatment temperature and its effect on photocatalytic activity: when the heat treatment temperature is between 450 and $650^{\circ} \mathrm{C}$, a pairing effect occurs. When the heat treatment temperature is higher than $650^{\circ} \mathrm{C}$, the photocatalytic activity of nano- $\mathrm{TiO}_{2}$ decreases significantly. When the heat treatment temperature reaches $950^{\circ} \mathrm{C}$, the removal rate of $\mathrm{TiO}_{2}$ to the methyl orange aqueous solution is almost zero. This is because, when the calcination temperature is low, the degree of crystallisation degree of anatase is low and the particle size of $\mathrm{TiO}_{2}$ is small, and the shorter the time required for the photogenic carrier to diffuse from the grain to the surface, the better the photogenic charge separation, and the lower the recombination probability of the electron and hole. On the other hand, the particle size of nano- $\mathrm{TiO}_{2}$ is smaller, the proportion of surface atoms increases and the light absorption efficiency is higher. In addition, due to the large specific surface area of the nanoparticles and the large contact area upon which the reaction can occur, it is also beneficial to the adsorption of the reactants, which leads to its high photocatalytic activity (Cheng et al. 2016,

Table 3: Apparent activation energies, temperature of fastest grain growth, and crystalline phase transformation of samples.

\begin{tabular}{|llllll|}
\hline Samples & $T_{\text {trans }} /{ }^{\circ} \mathrm{C}$ & $T_{\text {fast }} /{ }^{\circ} \mathrm{C}$ & & $E_{\mathrm{a}} / \mathrm{kJ} . \mathrm{mol}^{-1}$ \\
\cline { 2 - 5 } & $\mathrm{XRD}$ & $\mathrm{DYN}$ & $T_{\mathrm{a}}$ & $T_{\mathrm{r}}$ & \\
\hline $\mathrm{TiO}_{2}$ & 730 & & 546 & 1280 & 18.15 \\
\hline
\end{tabular}




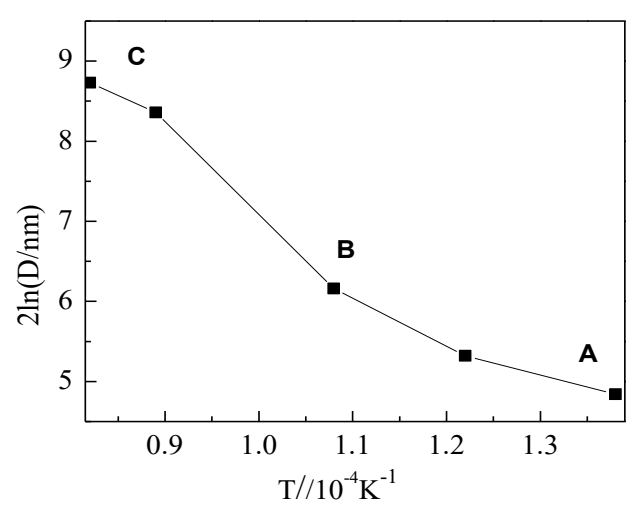

Fig. 4: Relationship between $2 \ln D$ and $1 / T$.

Sofianea \& Bilel 2016). When calcined at $650^{\circ} \mathrm{C}$, the anatase crystal type developed completely, while anatase-type $\mathrm{TiO}_{2}$ was mixed with a small amount of rutile-type $\mathrm{TiO}_{2}$, and the removal of methyl orange by photocatalytic degradation reached $97.75 \%$ (the photocatalytic activity of the sample prepared in this study, under the same conditions, had a $\mathrm{P}_{25}$ value for methyl orange removal of up to $98.63 \%$ ).

XRD analysis shows that $\mathrm{P}_{25}$ also contained two crystal types (with $650^{\circ} \mathrm{C}$ calcined $\mathrm{TiO}_{2}$ being similar therewith). This kind of mixed crystal $\mathrm{TiO}_{2}$ is not a simple combination of anatase and rutile, but a thin rutile crystal layer grows on the surface of anatase crystal, forming the so-called "mixed crystal effect", which not only does not hinder the absorption of light by the "nucleus", but also because of the rutile $\mathrm{TiO}_{2}$ having a narrow bandgap $\left(E_{\mathrm{g}}=3.0 \mathrm{eV}\right)$, it is easily excited by lower-energy light (which has a longer wavelength and is closer to ultraviolet light), owing to the difference in the rutile and anatase band gaps, the hole migrates from the valence band of anatase to the surface rutile valence band, and the photogenerated electron migrates from the rutile conduction band of the surface to the anatase phase, which effectively promotes the separation of photogenerated electron-hole separation in anatase and rutile crystals and reduces the recombination of electron-hole pairs, thus improving the photocatalytic activity of the catalyst (Kuang et al. 2020, Arbuj et al. 2010, Yu et al. 2003).

As the temperature continues to rise, the amount of rutile phase continues to increase, $\mathrm{TiO}_{2}$ particles grow rapidly, and the specific surface area decreases. Furthermore, rutile-anatase high-temperature transition occurs, treatment factors caused by the difference in the surface state of the material arise, surface-active group reduction occurs (on the anatase-type $\mathrm{TiO}_{2}$ surface with catalytically active groups $\mathrm{OH}^{-}$and $\mathrm{Ti}^{3+}$ ), the $\mathrm{TiO}_{2}$ surface undergoes a dehydrogenation reaction and the surface hydroxyl groups are lost, whereas hydroxyl groups can capture the photogenerated holes to form

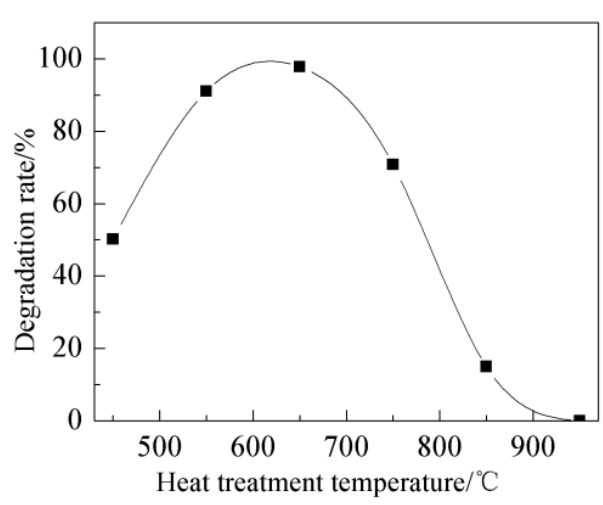

Fig. 5: The influence of sintering temperature on catalytic activity.

highly-oxidised hydroxyl radicals $(\cdot \mathrm{OH})$. In addition, rutile $\mathrm{TiO}_{2}$ has poor adsorption capacity for $\mathrm{O}_{2}$, a lower specific surface area, and photogenic electrons and holes are easily compounded. Moreover, the rutile type material has a low ability to adsorb oxygen, and photo-generated electrons are difficult to remove therefrom, which significantly reduces its photocatalytic performance (Xiao et al. 2010).

\section{CONCLUSIONS}

$\mathrm{TiO}_{2}$ was prepared by sol-gel method, and the effects of heat treatment temperature on $\mathrm{TiO}_{2}$ crystal structure, particle size, and photocatalytic performance were investigated. In addition, the phase transition of grains was analysed from the perspective of reaction kinetics. The results show that, with increasing calcination temperature, $\mathrm{TiO}_{2}$ crystallisation was gradually completed, the grains gradually grew in size, and at 450 to $550^{\circ} \mathrm{C}$, a thermally stable region was reached, the energy was mainly used for atomic rearrangement, reducing both the number and size of grain boundary defects and reducing the grain boundary free energy, so the particle size increase was small; beyond $650^{\circ} \mathrm{C}$, the energy was used for grain boundary migration, and the grains grew rapidly: some grains in the sample were transformed from an anatase phase to a rutile phase. The rate of degradation of methyl orange solution reached a maximum when calcined at $650{ }^{\circ} \mathrm{C}$ for $2 \mathrm{~h}$. At this time, $\mathrm{TiO}_{2}$ contained mixed anatase and rutile phases; this indicated that the mixed crystal effect of $\mathrm{TiO}_{2}$ can effectively inhibit photogenic electron-hole recombination and that the sample has a better photocatalytic performance than single anatase- $\mathrm{TiO}_{2}$.

\section{ACKNOWLEDGEMENTS}

This work was financially supported by the Natural Science Foundation of China (Grant No. 50708037), the National Science Fund for Excellent Young Scholars of China (Grant No. 51522402), and the Fund for Science and 
Technology Research Projects of Henan Province (Grant No. 182102311080).

\section{REFERENCES}

Al-Mamuna, M. R., Kaderb, S., Islam, M. S. and Khan, M. Z. H. 2019. Photocatalytic activity improvement and application of $\mathrm{UV}-\mathrm{TiO}_{2}$ photocatalysis in textile wastewater treatment: A review. J. Environ. Chem. Eng., 7(5): 1-17.

Amora, C. O., Elghnijia, K., Virlan, C. T., Pui, A. and Elaloui, E. 2019. Effect of dysprosium ion $\left(\mathrm{Dy}^{3+}\right)$ doping on morphological, crystal growth and optical properties of $\mathrm{TiO}^{2}$ particles and thin films. Physica B: Condens. Matter, 560: 67-74.

Arbuj, S. S., Hawaldar, R. R., Mulik, U. R., Wani, B. N., Amalnerkar, D. P. and Waghmode, S. B. 2010. Preparation, Characterization and photocatalytic activity of $\mathrm{TiO}_{2}$ towards methylene blue degradation. Mater. Sci. Eng.: B, 168(1/2/3): 90-94.

Chekem, C. T., Goetz, T., Richardson, Y., Richardsonb, Y., Plantarda, G. and Blinc, J. 2019. Modelling of adsorption/photodegradation phenomena on $\mathrm{AC}-\mathrm{TiO}_{2}$ composite catalysts for water treatment detoxification. Catal. Today, 328(15): 183-188.

Cheng, P. F., Wang, Y. L., Xu, L. P., Sun, P., Su, Z. S., Jin, F. M., Liu, F. M., Sun, Y. F. and Lu, G. Y. 2016. High specific surface area urchinlike hierarchical $\mathrm{ZnO}-\mathrm{TiO}_{2}$ architectures: Hydrothermal synthesis and photocatalytic properties. Mater. Lett., 175: 52-55.

Daniyal, M., Akhtar, S. and Azam, A. 2019. Effect of nano- $\mathrm{TiO}_{2}$ on the properties of cementitious composites under different exposure environments. J. Mater. Res. Technol., 8(6): 6158-6172.

Hakki, H. K., Allahyari, S., Rahemi, N. and Tabihi, M. 2019. Surface properties, adherence, and photocatalytic activity of sol-gel dip-coated $\mathrm{TiO}_{2}-\mathrm{ZnO}$ films on glass plates. Comptes Rendus Chimie, 22(5): 392-405.

Hu, Y. L., Liu, H. F. and Guo, X. P. 2010. Effect of water consumption on preparation of nitrogen-doped nanometer titanium dioxide by sol-gel method. J. Silic., 38(1): 97-104.

Jithin, M., Saravanakumar, K., Ganesan, V., Reddy, V.R., Razad, P. M., Patidar, M. M., Jeyadheepan, K., Marimuthu, G., Sreelakshmi, V. R. and Mahalakshmi, K. 2017. Growth, mechanism and properties of $\mathrm{TiO}_{2}$ nanorods embedded nanopillar: Evidence of lattice orientation effect. Superlattices and Microstruct., 109:145-153.

Kuang, J. Y., Xing, Z. P., Yin, J. W., Li, Z. Z., Tan, S. Y., Li, M., Jiang, J. J., Zhu, Q. and Zhou, W. 2020. $\mathrm{Ti}^{3+}$ self-doped rutile/anatase/TiO $(\mathrm{B})$ mixed-crystal tri-phase heterojunctions as effective visible-light-driven photocatalysts. Arab. J. Chem., 13: 2568-2578.

Li, Y., Ding, M. J. and Li, R. 2018. Hydrothermal preparation of claybased $\mathrm{TiO}_{2}$ and photocatalytic property thereof. Inorganic Chem. Ind., 50(12): 75-82.
Liao, Y. L., Que, W. X., Tang, Z. H., Wang, W. J. and Zhao, W. H. 2011. Effects of heat treatment scheme on the photocatalytic activity of $\mathrm{TiO}_{2}$ nanotube powders derived by a facile electrochemical process. J. Alloys and Compd., 509: 1054-1059.

Liu, Z.C., Zheng J. T. and Zhao, D. F. 2013. Metal ion doping modified nano- $\mathrm{TiO}_{2}$ band structure and photocatalytic properties. Silic. J., 41(3): 402-408.

Reddy, P. L., Kavitha, B., Reddy, P. A. K. and Kim, K. H. 2017. TiO photocatalytic disinfection of microbes in aqueous media: A review. Environ. Res., 154: 296-303.

Sofianea, S. and Bilel, M. D. 2016. Effect of specific surface area on photoelectrochemical properties of $\mathrm{TiO} 2$ nanotubes, nanosheets and nanowires coated with TiC thin films. J. Photochem. Photobiology A: Chem., 324: 126-133.

Srikanth, B., Goutham, R., Narayan, R. B., Ramprasath, A., Gopinath, K.P. and Sankaranarayanan, A.R. 2017. Recent advancements in supporting materials for immobilised photocatalytic applications in waste water treatment. J. Environ. Manage., 200: 60-78.

Sun, M. L., Qian, H. Y. and Chen, J. 2017. Preparation and photocatalytic performance of $\mathrm{TiO}_{2}$ thin films. Bull. Chin. Ceram. Soc., 36(8): 25952599.

Tong, Z., Wang, J. Y. and Fan, L. 2018. Effect of heat treatment on $\mathrm{TiO}_{2}$ thin film and it's photocatalytic performance. J. Synth. Crystals, 47(9): 1764-1769.

Varnagiris, S., Medvids, A., Lelis, M., Milciusa, D. and Antuzevics. A. 2019. Black carbon-doped $\mathrm{TiO}_{2}$ films: Synthesis, characterization and photocatalysis. J. Photochem. Photobiology A: Chem., 382(1): 1-9.

Wei, X., Cai, H. D., Feng, Q. G., Liu, Z., Ma, D. C., Chen, K. and Huang, Y. 2018. Synthesis of co-existing phases $\mathrm{Sn}-\mathrm{TiO} 2$ aerogel by ultrasonicassisted sol-gel method without calcination. Mater. Lett., 228(1): 379-383.

Xiao, N., Li, Z., Liu, J. W. and Gao, Y. 2010. Effects of calcination temperature on the morphology, structure and photocatalytic activity of titanate nanotube thin films. Thin Solid Films, 519(1): 541-548.

Yu, J. G., Yu, H. G., Cheng, B., Zhao, X. J., Yu, J. C. and Ho, W. K. 2003. The effect of calcination temperature on the surface microstructure and photocatalytic activity of $\mathrm{TiO}_{2}$ thin films prepared by liquid phase deposition. J. Phys. Chem. B, 107(50): 13871-13879.

Zarhri, Z. K., Cardos, M. A. A., Ziat, Y. N., Maryama, H., Omar, E. R., Julio, C. C. A. and David, A. A. 2020. Synthesis, structural and crystal size effect on the optical properties of sprayed $\mathrm{TiO}_{2}$ thin films: Experiment and DFT TB-MBJ. J. Alloys Compd., 819: 1-6.

Zhang, Y. L., Yang, J. and Yu, X. J. 2017. Preparation, characterization, and adsorption- photocatalytic activity of nano $\mathrm{TiO}_{2}$ embedded in diatomite synthesis. Rare Met., 991-987:(12)36.

Zhang, Y. L., Zhao, C. and Wu, C. 2014. $\mathrm{Zn}^{2+}$ preparation and properties of doped titanium dioxide composite films. Journal of IOL, 43(1): 19-24. 